\title{
State feedback linearization using block companion similarity transformation
}

\author{
Kessal Farida, Hariche Kamel, Bentarzi Hamid, Boushaki Razika \\ Signals and Systems Laboratory, Institute of Electrical and Electronic Engineering, University M'hamed BOUGARA of \\ Boumerdes, Algeria
}

\begin{tabular}{|c|c|}
\hline Article Info & ABSTRACT \\
\hline Article history: & In this research work, a new method is proposed for linearizing a class of \\
\hline Received Sep 17, 2020 & $\begin{array}{l}\text { nonlinear multivariable system; where the number of inputs divides exactly } \\
\text { the number of states. The idea of proposed method consists in representing }\end{array}$ \\
\hline Revised Nov 18, 2020 & the original nonlinear system into a state-dependent coefficient form and \\
\hline Accepted Dec 5, 2020 & $\begin{array}{l}\text { applying block similarity transformations that allow getting the linearized } \\
\text { system in block companion form. Because the linearized system's }\end{array}$ \\
\hline Keywords: & $\begin{array}{l}\text { eigenstructure can determine system performance and robustness far more } \\
\text { directly and explicitly than other indicators, the given class multivariable }\end{array}$ \\
\hline $\begin{array}{l}\text { Block companion form } \\
\text { Eigenstructure }\end{array}$ & $\begin{array}{l}\text { system is chosen. Examples are used to illustrate the application and show } \\
\text { the effectiveness of the given approach. }\end{array}$ \\
\hline
\end{tabular}

Feedback linearization

Multivariable nonlinear system

Similarity block transformation

This is an open access article under the CC BY-SA license.

\section{Corresponding Author:}

Kessal Farida

Signals and Systems Laboratory

Institute of Electrical and Electronic Engineering

University M'hamed BOUGARA of Boumerdes, Algeria

Email: f.kessal@univ-boumerdes.dz

\section{INTRODUCTION}

The feedback linearization technique is one of modern tools, which allows synthesizing a control law for smooth, continuous, non-linear systems [1-5]. The basic idea is to transform a nonlinear system into a linear one via nonlinear change of coordinates only (state linearization problem) or by nonlinear feedback and change of coordinates (feedback linearization problem) so that the linear control techniques can be applied [1]. Feedback linearization has been one of the most active research topics in recent years; linearization of affine systems [6], systems with nonsmooth nonlinearities [7], exact linearization [8]. Various methods exist to find feedback linearizable form for single input [9], and multi-input nonlinear systems [10-15].

Significant research effort has been devoted to the construction of approximate solutions to the problem of linearizing nonlinear systems by state or output feedback. Main reason for that is the limited applicability of the rigorous methods, and the complexity, sensitivity and design difficulties of the exact linearizing compensators, if any [16]. The analysis and the comparison of approximate linearization and exact linearization are proposed by [17]. If the original nonlinear system cannot be linearized exactly by state feedback, the method of approximate feedback linearization [16, 18-22] is used. This process consists of finding approximate output functions that satisfy the involutivity condition up to determined system order, $[16,19]$ adopted a two-step procedure to solve the approximate linearization; first a state transformation matrix is settled, so that the nonlinear system is transformed approximately into the controllable canonical form. Second, a standard nonlinear linearization method is used to transform the controllable canonical form into a stable linear system. 
Kabanov [19] offered an approach that consists in representing the original nonlinear (single and multivariable) system into a state-dependent coefficient form and applying similarity transformations that allow getting the system to canonical form, that considerably simplifies the problem of feedback linearization afterwards. Such similarity transformations allow accomplishing linearization of system without considering the virtual output function $y=h(x)$ [19].This work consists in applying a block companion similarity transformation to a class of nonlinear multivariable system; where the number of inputs divides exactly the number of states. It is known that if a linear-time-invariant MIMO system described by a state space equation has a number of states divisible by the number of inputs and it can be transformed to block controller form, we can design a state feedback controller using block pole placement technique by assigning a set of desired Block poles. These may be left or right block poles [23-26]. The eigenvalues and eigenvectors (eigenstructure) of the state matrix can determine system performance and robustness more directly and explicitly than other indicators. Hence their assignment should improve feedback system performance and robustness distinctly and effectively [25].

In the present work, firstly we have started by introducing feedback linearization used in multivariable systems. As a second section a problem of transforming a multivariable system into block controller form is formulated, it is then followed by the discussion of the feedback linearization using similarity block transformation for the class of MIMO system which is the heart of this work. In a forth section examples are used to show different steps and the effectiveness of the proposed method, and finally the paper is finished by a conclusion.

\section{PROBLEM FORMULATION}

Consider a class of multivariable nonlinear system

$$
\begin{aligned}
& \dot{x}=f(x)+\sum_{i=1}^{m} g_{i}(x) u_{i} \\
& \dot{x}=f(x)+G(x) u \\
& y_{i}=h_{i}(x), i=1, \ldots, m \\
& \text { or } Y=H(x)
\end{aligned}
$$

where $f, g$ and $\mathrm{h}$ are sufficiently smooth, $m$ is the number of inputs and outputs, $n$ is the number of states. The multivariable system (1), in the state-dependent coefficient form is given by:

$$
\dot{x}(t)=A(x) x+B(x) u
$$

where $A(x)$ is an $\times n$ and $B(x)$ is an $n \times m$ matrices respectively; are continuous differentiable.

Consider the system (1), the feedback linearization problem consists in finding the nonsingular coordinate transformation $z=T_{F L}(x)$ and the input control law $u=u_{F L}$ such that the system (1) can be transformed into linear block canonical form. Under condition of the existence of smooth function $H(x)$, transformation $z=T_{F L}(x)$ is described by (1).

$$
T_{F L}(x)=\left[\begin{array}{c}
H(x) \\
L_{f}^{1} H(x) \\
\vdots \\
L_{f}^{l-1} H(x)
\end{array}\right]
$$

and linearizing control is given as,

$$
u_{F L}=s^{-1}(x)(v-q(x))
$$

where $q(x)=L_{f}^{l} H(x)$ and $s(x)=L_{G} L_{f}^{l-1} H(x)$ and $v$ is the new control input; $L_{f}^{i} H(x)$ is the Lie derivative of the $i^{\text {th }}$ order from the function $H(x)$ along the vector field $f[1,19]$.

Applying feedback linearizing control $u_{F L}$ (4), in respect of the transformation $T_{F L}(x)$ (3), the system (1) has the following form,

$$
\dot{z}=A_{c} z+B_{c} v
$$


where $\left(A_{c}, B_{c}\right)$ is in block canonical form.

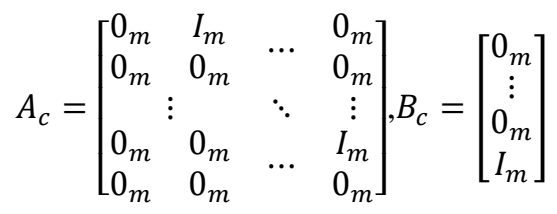

where $0_{m}$ and $I_{m}$ are $m \times m$ zero and identity matrices, respectively.

Applicability of the normal form method for feedback linearization is ensured by the existence of diffeomorphism (3) and observance of the terms [1, 19],

$$
\begin{aligned}
& L_{G} H(x)=L_{G} L_{f}^{1} H(x)=\ldots=L_{G} L_{f}^{l-2} H(x)=0_{m} \\
& s(x)=L_{G} L_{f}^{l-1} H(x) \neq 0_{m}
\end{aligned}
$$

which is not always true. In such situations, as a rule, methods of approximate feedback linearization are used $[16,19,27]$. The block canonical similarity transformation used for feedback linearizing control design is:

$$
z=T(x) x, x=T^{-1}(x) z, T(x) \in \mathcal{R}^{m x n}
$$

\section{FEEDBACK LINEARIZATION}

System (1) can be represented in the state dependent coefficient form [28]:

$$
\dot{x}(t)=A(x) x+B(x) u
$$

Let us assume that matrix $A(x)$ is $n \times n$ and vector $B(x)$ is, $n \times m$ is continuously differentiable. It is also supposed that the pair $(A(x), B(x))$ is a full block controllable, i.e. for the system (8) controllability matrix has full rank (rank of $w(x)=l$ ), for $t \geq t_{0}$.

$$
w(x)=\left[B(x) \quad A(x) B(x) \quad \ldots \quad A^{l-1}(x) B(x)\right]
$$

where $\frac{n}{m}=l, l$ (an integer) is the number of blocks.

Applying transformation (7) to (8) we get (5) for which the following relations are true.

$$
\begin{aligned}
& \dot{z}(t)=\dot{T}(x) x+T(x) \dot{x} \\
& \dot{z}(t)=\dot{T}(x) T^{-1}(x) z+T(x)\left[A(x) T^{-1}(x) z+T(x) B(x) u\right] \\
& \dot{z}(t)=A_{c}(z) z+B_{c}(z) u(z) \\
& A_{c}(z)=T(x) A(x) T^{-1}(x)+\dot{T}(x) T^{-1}(x)
\end{aligned}
$$

where

$$
\begin{aligned}
A_{c} & =\left[\begin{array}{cccc}
0_{m} & I_{m} & \ldots & 0_{m} \\
\vdots & \vdots & \ddots & \vdots \\
\vdots & \vdots & \ddots & I_{m} \\
-A_{0}(z) & -A_{1}(z) & \ldots & -A_{l-1}(z)
\end{array}\right] \\
B_{c} & =T(x) B(x)=\left[\begin{array}{c}
0_{m} \\
\vdots \\
0_{m} \\
I_{m}
\end{array}\right]
\end{aligned}
$$

where $A_{i} \in \mathfrak{R}^{m x m}, i=1,2, \ldots, . l . \mathrm{I}_{\mathrm{m}}$ and $0_{m}$ are $m \times m$ identity and null matrices respectively, $l$ is an integer such that $l=\frac{n}{m}$ where $n$ is the order of the system and $m$ is the number of inputs. 
The right matrix fraction description (RMFD) [24-26] of the system can be formulated directly from (11-a) as,

$$
F(\lambda)=N_{R}(\lambda) D_{R}^{-1}(\lambda)
$$

where,

$$
\begin{aligned}
& D_{R}(\lambda)=I_{m} \lambda^{l}+A_{1} \lambda^{l-1}+\ldots+A_{l} \\
& N_{R}(\lambda)=I_{m} \lambda^{l}+C_{1} \lambda^{l-1}+\ldots+C_{l}
\end{aligned}
$$

$C_{i} \in \mathcal{R}^{m x m}$ are constant matrices. The desired matrix polynomial $A_{D}(\lambda)$ if $R_{i}$ is a right solvent is given by,

$$
\begin{aligned}
& A_{D}(\lambda)=R_{i}^{l}+D_{1} R_{i}^{l-1}+\cdots+D_{l} \\
& {\left[\begin{array}{llll}
D_{d l} D_{d(l-1)} & \ldots . & D_{d 1}
\end{array}\right]=-\left[\begin{array}{llll}
R_{1}^{l} R_{2}^{l} & \ldots & R_{l}^{l}
\end{array}\right] V_{R}^{-1}}
\end{aligned}
$$

where $V_{R}$ is the right block Vandermonde matrix[24, 25]. An alternative factorization of $F(\lambda)$ is the left matrix fraction description (LMFD) defined by the following equation;

$$
F(\lambda)=D_{L}^{-1}(\lambda) N_{L}(\lambda)
$$

where $D_{L}(\lambda)$ is the left denominator (13) and $N_{L}(\lambda)$ is the left numerator (14). The desired matrix polynomial $A_{D}(\lambda)$ if $\mathrm{L}_{\mathrm{i}}$ is a left solvent is:

$$
A_{D}(\lambda)=L_{i}^{l}+L_{i}^{l-1} D_{1}+\cdots+D_{l}
$$

the desired solvents are given as,

$$
\left[\begin{array}{c}
D_{d l} \\
D_{d(l-1)} \\
\vdots \\
D_{d 1}
\end{array}\right]=-\left[\begin{array}{cccc}
I_{m} & L_{1} & \ldots & L_{1}^{l-1} \\
I_{m} & L_{2} & \ldots & L_{2}^{l-1} \\
\vdots & \vdots & \ddots & \vdots \\
I_{m} & L_{l} & \ldots & L_{l}^{l-1}
\end{array}\right]^{-1}\left[\begin{array}{c}
L_{1}^{l} \\
L_{2}^{l} \\
\vdots \\
L_{l}^{l}
\end{array}\right]
$$

Linearizing control for block companion system (11) can be defined in the form of the feedback;

$$
u(z)=u_{F L}(z)+v(z)=K(z) z+G_{c}(z) z
$$

where matrix $G_{c}(z)$ can be calculated, for instance, by means of the method of the placement of the closedloop system block poles. $K(z)$ is computed by $[19,21]$.

$$
K(z)=-\left[A_{l-1}(z) \quad A_{l-2}(z) \quad \ldots \quad A_{0}(z)\right]
$$

In terms of initial system state variables the linearizing control (16) can be written as,

$$
u(x)=u_{F L}(x)+v(x)=K(x) T(x) x+G_{c} T
$$

The procedure to find the coordinate of change is $[24,25]$ :

$$
T(x)=\left\{T_{i}(x)\right\}, i=1, \ldots, l
$$

let

$$
T_{c 1}=\left[\begin{array}{llll}
0_{m} & 0_{m} & \cdots & I_{m}
\end{array}\right] w^{-1}(x)
$$




$$
T(x)=\left[\begin{array}{c}
T_{c 1} \\
T_{c 1} A \\
\vdots \\
T_{c 1} A^{l-1}
\end{array}\right]
$$

where $0_{m}$ and $I_{m}$ are null and identity matrices, respectively. The transformation (7), (19) exists, if and only if:

- controllability matrix $w(x)$ has full rank $n$ at $t \geq t_{0}$

$-\frac{n}{m}=l, l$ is an integer

If we suppose that the block canonical form has the form (6).

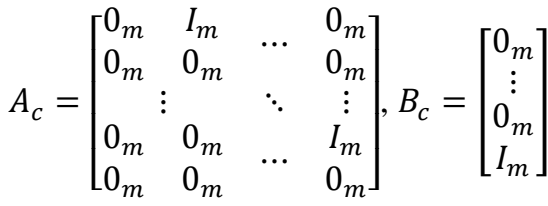

$$
\begin{aligned}
& \text { i.e. } K(z)=\left[\begin{array}{lll}
0_{m} & \ldots & 0_{m}
\end{array}\right]
\end{aligned}
$$

The linearizing control is:

$$
u(z)=v(z)=G_{c}(z) z=K_{c}(z) z
$$

In the next section, numerical examples are illustrated to show the effectiveness of the proposed method.

\section{ILLUSTRATIVE EXAMPLES}

\subsection{Example 1}

Let consider the following nonlinear multivariable system, with a number of states is $n=3$ and a number of inputs is $m=3$ therefore a number of blocks is $l=\frac{3}{3}=1$. The control problem is to stabilize the system (22) by means of the feedback.

$$
\begin{aligned}
& \dot{x}_{1}=x_{1}+x_{2}^{2}+u_{1} \\
& \dot{x}_{2}=-x_{1}+2 x_{3}+2 u_{2} \\
& \dot{x}_{3}=\left(x_{1}-1\right) x_{2}+u_{3}
\end{aligned}
$$

where,

$$
f(x)=\left[\begin{array}{c}
x_{1}+x_{2}^{2} \\
-x_{1}+2 x_{3} \\
\left(x_{1}-1\right) x_{2}
\end{array}\right] \text { and } g(x)=\left[\begin{array}{c}
u_{1} \\
2 u_{2} \\
u_{3}
\end{array}\right]
$$

We write $f(x)$ and $g(x)$ in state-dependent coefficient form; $A(x)$ and $B(x)$, respectively.

$$
A(x)=\left[\begin{array}{ccc}
1 & x_{2} & 0 \\
-1 & 0 & -2 \\
x_{2} & -1 & 0
\end{array}\right], B(x)=\left[\begin{array}{lll}
1 & 0 & 0 \\
0 & 2 & 0 \\
0 & 0 & 1
\end{array}\right]
$$

We compute the similarity transformation matrix.

$$
w(x)=[B(x)]=\left[\begin{array}{lll}
1 & 0 & 0 \\
0 & 2 & 0 \\
0 & 0 & 1
\end{array}\right]
$$

The rank of $w(x)$ is 3 (full rank), hence the system is controllable. Now we compute the coordinate of change $T(x)$ we calculate first. 


$$
T_{c 1}=\left[I_{3}\right] w^{-1}(x)
$$

then,

$$
T(x)=\left[T_{c 1}\right]=\left[\begin{array}{lll}
1 & 0 & 0 \\
0 & \frac{1}{2} & 0 \\
0 & 0 & 1
\end{array}\right]
$$

hence,

$$
\begin{aligned}
& A_{c}(z)=T(x) A(x) T^{-1}(x) \\
& A_{c}(z)=\left[\begin{array}{ccc}
1 & 2 x_{2} & 0 \\
-\frac{1}{2} & 2 & -1 \\
x_{2} & -2 & 0
\end{array}\right]
\end{aligned}
$$

and $K(z)=-A_{c}(z)$, and we have

$$
B_{c}(z)=T(x) B(x)=\left[\begin{array}{lll}
1 & 0 & 0 \\
0 & 1 & 0 \\
0 & 0 & 1
\end{array}\right]
$$

In this application, $G_{c}(z)$ is computed using desired block poles of the closed loop system. The eigenvalues of close-loop system matrix are $\lambda_{1}=-3, \lambda_{2}=-4$ and $\lambda_{3}=-5$. The characteristic matrix polynomial of the closed-loop system is forced to equal a desired matrix polynomial of the form.

$$
A_{D}(\lambda)=I_{m} \lambda^{l}+D_{1} \lambda^{l-1}+\cdots+D_{l}
$$

The desired characteristic matrix polynomial $A_{D}(\lambda)$ may be constructed from a set of desired $m \times m$ block poles or matrix roots of $A_{D}(\lambda)$; These block poles can be constructed under different forms either an $m \times m$ diagonal form matrix, an $m \times m$ controller canonical form matrix or an $m \times m$ observer canonical matrix. Using block poles in diagonal form gives a better response, robustness and smaller sensitivities [24], for this reason only the case of the diagonal form will be studied. Feedback gain of the closed-loop system $G_{c}$ is computed as:

$$
A-B G_{c}=A_{D}
$$

$A_{D}$ is a desired closed-loop matrix in block diagonal form where each block is constructed from desired eigenvalues. The desired block pole constructed in diagonal form is given by,

$$
R=\left[\begin{array}{ccc}
-3 & 0 & 0 \\
0 & -4 & 0 \\
0 & 0 & -5
\end{array}\right]
$$

The corresponding $3 \times 3$ desired right denominator matrix polynomial of degree 1 is:

$$
D_{f}(s)=I s+D_{f 1} \text { where } D_{f 1}=-R^{2} V_{R}^{-1} \text { i.e., } D_{f 1}=\left[\begin{array}{lll}
3 & 0 & 0 \\
0 & 4 & 0 \\
0 & 0 & 5
\end{array}\right]
$$

Now we can find $G_{c}$

$$
G_{c}=\left[\begin{array}{ccc}
3 & 2 x_{2} & 0 \\
-1 / 2 & 4 & -1 \\
x_{2} & -2 & 5
\end{array}\right]
$$

The linearizing control.

$$
u(z)=u_{F L}(z)+v(z)=K(z) z+G_{c}(z) z=\left[K(z)+G_{c}(z)\right] z=K_{c}(z) z
$$


where

$$
K c=\left[\begin{array}{ccc}
-3 & 0 & 0 \\
0 & -4 & 0 \\
0 & 0 & -5
\end{array}\right]
$$

its norm $\left\|K_{c}\right\|_{2}=5$. In the terms of initial system state variables linearizing control (18) can be written as,

$$
u(x)=K(x) T(x) x+G_{c}(x) T(x) x=\left(K(x)+G_{c}(x)\right) T(x) x=K_{c}(x) T(x) x
$$

hence,

$$
u(x)=\left[\begin{array}{ccc}
-3 & 0 & 0 \\
0 & -4 & 0 \\
0 & 0 & -5
\end{array}\right]
$$

The results of the nonlinear system (22) with the linearizing control (32), For modeling the following initial values were taken: $x_{1}(0)=0, x_{2}(0)=1, x_{3}(0)=-1$. Using MATLAB software (M. file), the state variables responses are shown in this Figure 1. The results of the figure demonstrate that the system is stable.

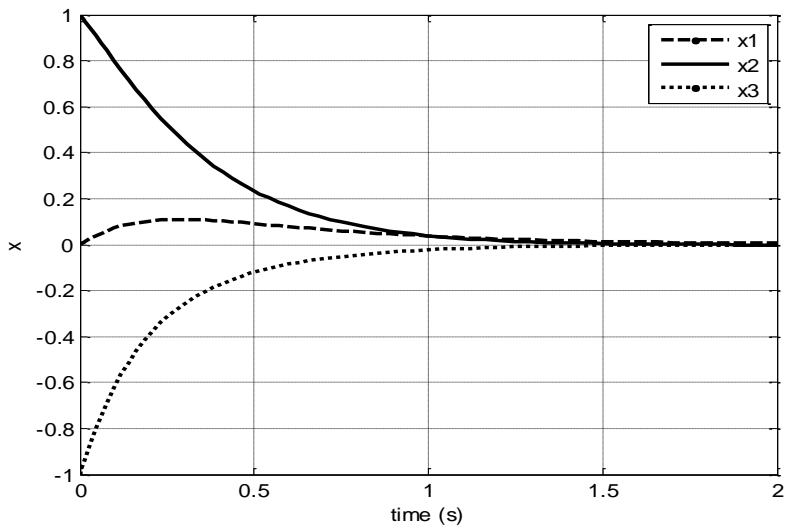

Figure 1. State variables result for the system (22)

\subsection{Example 2}

In the following nonlinear multivariable system, a number of states is $n=4$ and a number of inputs is $m=2$ therefore a number of blocks is $l=\frac{4}{2}=2$. The control problem is to stabilize the system (33) by means of the feedback.

$$
\begin{aligned}
& \dot{x}_{1}=x_{1}+x_{2}+x_{3}^{2} \\
& \dot{x}_{2}=-x_{1}-2 x_{3}+u_{1} \\
& \dot{x}_{3}=x_{4} \\
& \dot{x}_{4}=\left(x_{1}-1\right) x_{2}+u_{2}
\end{aligned}
$$

where,

$$
f(x)=\left[\begin{array}{c}
x_{1}+x_{2}+x_{3}^{2} \\
-x_{1}-2 x_{3} \\
x_{4} \\
\left(x_{1}-1\right) x_{2}
\end{array}\right] \text { and } g(x)=\left[\begin{array}{c}
0 \\
u_{1} \\
0 \\
u_{2}
\end{array}\right]
$$

We write $f(x)$ and $g(x)$ in state-dependent coefficient form; $A(x)$ and $B(x)$, respectively.

$$
A(x)=\left[\begin{array}{cccc}
1 & 1 & x_{3} & 0 \\
-1 & 0 & -2 & 0 \\
0 & 0 & 0 & 1 \\
x_{2} & -1 & 0 & 0
\end{array}\right], B(x)=\left[\begin{array}{ll}
0 & 0 \\
1 & 0 \\
0 & 0 \\
0 & 1
\end{array}\right]
$$


We compute the block similarity transformation matrix.

$$
w(x)=[B(x) A(x) B(x)]=\left[\begin{array}{cccc}
0 & 0 & 1 & 0 \\
1 & 0 & 0 & 0 \\
0 & 0 & 0 & 1 \\
0 & 1 & -1 & 0
\end{array}\right]
$$

The rank of $w(x)$ is 4 (full rank), hence the system is controllable. Now we compute the coordinate of change $T(x)$, we compute first.

$$
T_{c 1}=\left[\begin{array}{llll}
0 & 0 & 1 & 0 \\
0 & 0 & 0 & 1
\end{array}\right] w^{-1}(x)
$$

then,

$$
T(x)=\left[\begin{array}{c}
T_{c 1} \\
T_{c 1} A
\end{array}\right]=\left[\begin{array}{cccc}
1 & 0 & 0 & 0 \\
0 & 0 & 1 & 0 \\
1 & 1 & x_{3} & 0 \\
0 & 0 & 0 & 1
\end{array}\right]
$$

hence,

$$
A_{c}(z)=T(x) A(x) T^{-1}(x)=\left[\begin{array}{cccc}
0 & 0 & 1 & 0 \\
0 & 0 & 0 & 1 \\
-1 & -2 & 1 & x_{3} \\
x_{2}+1 & x_{3} & -1 & 0
\end{array}\right]
$$

and we have

$$
B_{c}(z)=T(x) B(x)=\left[\begin{array}{ll}
0 & 0 \\
0 & 0 \\
1 & 0 \\
0 & 1
\end{array}\right]
$$

In this application, $G_{c}(z)$ is computed using desired block poles of the closed loop system. The eigenvalues of close-loop system matrix are $\lambda_{1}=-1, \lambda_{2}=-2$ and $\lambda_{3}=-3$ and $\lambda_{4}=-4$. The desired block pole constructed in diagonal form is given by,

$$
R_{1}=\left[\begin{array}{cc}
-1 & 0 \\
0 & -2
\end{array}\right] \text { and } R_{2}=\left[\begin{array}{cc}
-3 & 0 \\
0 & -4
\end{array}\right]
$$

hence, $\quad D_{f}(s)=I s^{2}+D_{f 1} s+D_{f 0}$

The linearizing control.

$$
u(z)=u_{F L}(z)+v(z)=K(z) z+G_{c}(z) z=\left[K(z)+G_{c}(z)\right] z=K_{c}(z) z
$$

where,

$$
K c=\left[\begin{array}{cccc}
2 & -2 & 5 & x_{3} \\
x_{2}+1 & x_{3}+8 & -1 & 6
\end{array}\right]
$$

In the terms of initial system state variables the linearizing control is.

$$
u(x)=\left[\begin{array}{cccc}
0.43 & 1.4 & 1.4 x_{3}-2 & x_{3} \\
x_{2} & -1 & 0.08 & 0.6
\end{array}\right]
$$

The results of the nonlinear system (33) with the linearizing control (41), for modeling the following initial values were taken: $x_{1}(0)=0, x_{2}(0)=1, x_{3}(0)=-1$ and $x_{4}(0)=-0.5$. Using MATLAB software (M. file), the state variables responses are shown in this Figure 2. The system is stable as it is indicated in the results of the figure. 


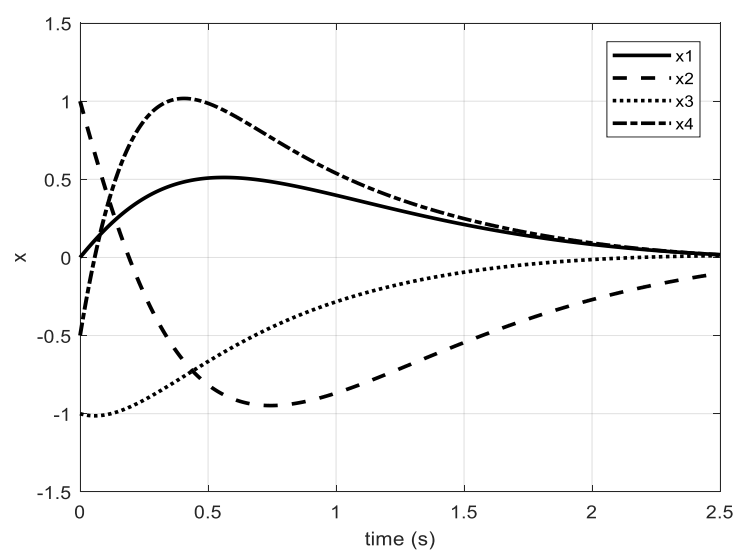

Figure 2. State variables result for the system (33)

\section{CONCLUSION}

In this work, state feedback linearization of a class of nonlinear multivariable systems; the number of states divides exactly the number of inputs, by using block canonical similarity transformation is presented. First the original nonlinear system is represented in state-dependent coefficient form then a block companion similarity transformation is applied to the system. Linearizing control for block canonical system is defined in the form of the feedback; where the feedback gain is computed by forcing the characteristic matrix polynomial of the closed-loop system to equal a desired matrix polynomial constructed from a set of desired block poles or matrix roots in diagonal form. In this approach some advantages are taken: i) Because there exists an approximate linearization method when the exact feedback linearization is not applicable, ii) Because using block controllable form, the eigenvalues and eigenvectors (eigenstructure) of the state matrix, can determine system performance and robustness, iii) Using block poles (solvents) in diagonal form gives a better time response, robustness and smaller sensitivities of the system, iv) Such block similarity transformations allow accomplishing linearization of system without considering the virtual output function $Y=H(x)$ or $y_{i}=h_{i}(x)$. In this work, similarity block transformation is developed for class of MIMO system, which seems to be a successful approach. As further work, an enhancement to the present method is proposed, the similarity block transformation will be applied to a general form of MIMO systems; for the case where $l$ (or $\frac{n}{m}$ ) is not an integer.

\section{REFERENCES}

[1] A. Isidori, "Nonlinear control systems,3rd ed,"New York: Springer Verlag, 1995.

[2] E. Aranda-Bricaire, C. H. Moog, and J.-B. Pomet, "A linear algebraic framework for dynamic feedback linearization, "IEEE Trans. Automat. Contr., vol. 40, no. 1, pp. 127-132, Jan. 1995.

[3] M. Vidyasagar, "Nonlinear systems analysis, 2nd Edition, "Prentice Hall, Englewood Cliffs, New Jersey, 1993.

[4] H. J. Marquez, "Nonlinear control systems analysis and design,"John Wiley \& sons, Apr 2003.

[5] H. K. Khalil, "Nonlinear systems, 3rd Edition," Prentice Hall, 2002.

[6] D. A. Fetisov,"On some approaches to linearization of affine systems," International Federation of Automatic Control, Elsevier, vol. 52, no. 16, pp. 700-705, 2019.

[7] S Jiffri, P Paoletti, and J E. Mottershead, "Feedback linearization in systems with nonsmooth nonlinearities," Journal of Guidance Control \& Dynamics, vol. 39, no. 4, pp. 814-825, 2016.

[8] A. Saito, K. Sekiguchi, and M. Sampei, "Exact linearization by time scale transformation based on relative degree structure of single-input nonlinear systems," IEEE Conf. on Decision and Control, pp. 5408-5413, 2010.

[9] I. A. Tall, "State and feedback linearizations of single-input control systems," Systems \& Control Letters, vol. 59, no. 7, pp. 429-441, 2010.

[10] T. Pengliang, "Linearization of MIMO nonlinear system with measurable disturbance," 12th International Conference on Measuring Technology and Mechatronics Automation, pp. 744-749, 2020.

[11] I. A. Tall, "explicit feedback linearization of control systems," 48th IEEE Conference on Decision and Control and 28th Chinese Control Conference Shanghai, P.R. China, pp. 7454-7459, 2009.

[12] A. Zawadzki and S. Różowicz, "Application of input- state of the system transformation for linearization of some nonlinear generators," Int. Journal of Control, Automation, and Systems, vol. 13, no. 3, pp. 626-633, 2015.

[13] S. Róhowicz and A. Zawadzki, "Input-output transformation using the feedback of nonlinear electrical circuits: algorithms and linearization examples," Hindawi, Mathematical Problems in Engineering, 2018.

[14] A. Ahmed, "Feedback linearization of RF power amplifier for TETRA standard," Bulletin of Electrical Engineering and informatics, vol. 3, no. 3, pp. 161-172, 2014. 
[15] W. Ghozlane and J. Knani, "Nonlinear control of MIMO system using feedback linearization control method and PD controller for tracking purpose," 5th International Conference on Control Engineering \& Information Technology. Proceeding of Engineering and Technology, vol. 32, pp. 86-90, 2017.

[16] G. O. Guardabassi and S. M. Savaresi, "Approximate linearization via feedback: an overview,"Automatica, vol. 37, no. 1 , pp. 1-15, 2001

[17] G. S. Cardosoand and L. Schnitman, "Analysis of exact linearization and aproximate feedback linearization techniques," Hindawi Publishing CorporationMathematical Problems in Engineering, 2011.

[18] K. Yamada, "Approximate feedback linearization for nonlinear systems and its application to the ACROBOT," Proceedings of the American Control Conference, Anchorage, AK, vol. 2, pp. 1672-1677, 2002.

[19] A. A. Kabanov, "Full-state linearization of systems via feedback using similarity transformation," International Siberian Conference on Control and Communications, pp. 1-5, 2016.

[20] A. Kabanov and V. Alchakov, "Composite control of nonlinear singularly perturbed systems via approximate feedback linearization," International Journal of Automation and Computing, pp. 1-11, 2017.

[21] C. Yang, S. Zheng, O. O. Peter, Q. Huang, and J. Han, "Approximate feedback linearization control for spatial 6DOF hydraulic parallel manipulator," The Open Mechanical Eng. Journal, vol. 5, no. 1, pp. 117-123, 2011.

[22] M. Sassano and A. Astolfi, "Approximate dynamic tracking and feedback linearization," IEEE 55th Conference on Decision and Control, pp. 5688-5693, 2016.

[23] K Hariche and E. D. Denman, "Interpolation theory and $\lambda$-matrices,"Journal of Mathematical Analysis and Applications, vol. 143,no. 2, pp. 530-547, 1989

[24] F. Kessal and K. Hariche, "On the choice of closed loop block poles in multi-variable control design," in Proc. Applications of Large Scale Industrial Systems, vol. 39, no. 14, pp. 59-63, 2006.

[25] M. Yaici and K. Hariche, "On eigenstructure assignment using block poles placement," European Journal of Control, vol. 20, no. 5, pp. 217-226, 2014.

[26] M. Yaici and K. Hariche, "On solvents of matrix polynomials," International Journal of Modeling and Optimization, vol. 4, no. 4, pp. 273-277, 2014.

[27] A. L. Fradkov, I. V. Miroshnik, and V. O. Nikiforov," Nonlinear and adaptive control of complex systems," Springer-Science+Bussines Media Dordrecht, 1999.

[28] Y. Sakayanagi, D. Nakayama, S. Nakaura, and M. Sampei, "Clarification of free parameters of state-dependent coefficient form: effect on solving state-dependent Riccati inequality," Proceedings of the 17th World Congress The International Federation of Automatic Control Seoul, Korea, vol. 41, no. 2, pp. 182-187, 2018.

\section{BIOGRAPHIES OF AUTHORS}

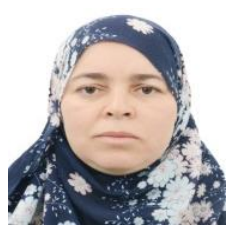

Kessal Farida is a teacher in Electrical Engineering at the university of Boumerdes (Algeria) in the Institute of Electrical and Electronic Engineering from 2007 to 2018, since 2019 in Tizi Ouzou university. She obtained Engineer Diploma (2001) at the university of Tizi ouzou, Algeria, magister Diploma (2006) at university of Boumerdes, since 2007 Doctorant in Automation.

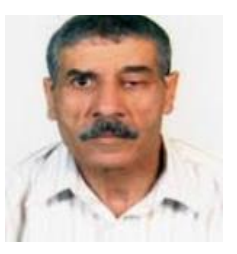

Hariche Kamel is a Professor at the Institute of Electrical and Electronics Engineering of Boumerdes (Algeria'). He obtained his mater degree in Control Engineering and $\mathrm{PhD}$ degree in 1978 and 1987, respectively, from the university of Huston in Texas. His research is related to linear and nonlinear systems as well as Automatic control of Multivariable Systems.

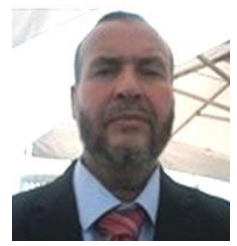

Bentarzi Hamid received both Electrical Engineering and Magister Degrees with honors in 1989 and 1992 respectively and Ph. D in Microelectronic systems in 2004. Till 1999, he was a lecturer at INELEC, Boumerdes, Algeria. Since 1999, he has been a faculty member at the Department of Electrical and Electronic Engineering, Faculty of Engineering, University of Boumerdes, Algeria. Since 2001, he has been heading of research team working in developing microelectronic systems applied to power systems in the Signal and System Laboratory, Boumerdes, Algeria. His current research interests are in the fields of microelectronics, electrical protection systems, electric energy systems and systems reliability. He has authored and coauthored over 50 technical papers. Besides, he has been a member of organizing and technical committee of several conferences including WSEAS group.

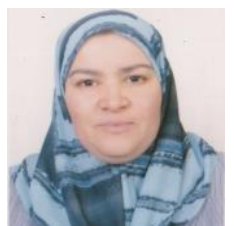

Boushaki Razika is a Teacher in Electrical Engineering at the university of Boumerdes (Algeria) in the Institute of Electrical and Electronic Engineering since 2003, she obtained Engineer Diploma (1995), magister Diploma (2003) at university of Boumerdes and Doctor (June 2013) in Electrical Engineering. She is member in the laboratory of the research since 2009. She introduced several practical automation systems in industry between 1999 and 2003.

State feedback linearization using block companion similarity transformation (Kessal Farida) 